\title{
Stress Distribution in Photoelastic Models of Transverse Sections of Porcelain-Fused-to-Gold Crowns and Preparations
}

\author{
R. G. CRAIG, M. K. EL-EBRASHI,* and J. W. FARAH
}

School of Dentistry, University of Michigan, Ann Arbor, Michigan 48104, USA

The stress distribution in composite photoelastic models of transverse sections of porcelain-fused-to-gold restorations and preparations was determined. The optimum design was one in which the porcelain-gold junction was on the lingual side of contact points that represented the contact of adjacent teeth.

The literature related to stress distribution in porcelain-fused-to-gold restorations and the supporting structures has been reviewed by Craig, El-Ebrashi, and Peyton. ${ }^{1}$ They used two-dimensional composite photoelastic models to study the stress distribution in labial-lingual and mesial-distal sections of a central incisor that contained a porcelainfused-to-gold or a porcelain crown restoration. The stress distribution was measured as a function of geometric design, position, and magnitude of loading. The various portions of the photoelastic models were constructed of various plastics so that the ratio of the elastic moduli was almost the same as that for porcelain, gold alloy, and human dentin. They found that the porcelain-togold joint near the shoulder should be $30^{\circ}$ from the horizontal and that porcelain supported by gold alloy was superior to a porcelain jacket crown. They established that the bulk of gold in the gingival region reduced the state of stress and that the porcelain-to-gold joint on the lingual shoulder should be as far as possible from the usual site of loading. They also found that com-

This investigation was supported, in part, by USPHS Research Grant DE-01817 from the National Institute for Dental Research, National Institutes of Health, Bethesda, Md.

Received for publication September 18, 1972.

* Present address: University of Cairo, School of Dentistry, Cairo, Egypt, UAR. plete coverage of the gold by porcelain was not superior to providing a bulk of gold alloy on the lingual shoulder.

In this study, a similar two-dimensional modeling procedure was used to study transverse sections through porcelain-fused-to-gold restorations. The geometry of the restorations and the magnitude of the loads were varied and the state of stress was determined by studying the isochromatic fringe patterns.

\section{Materials and Methods}

The geometry of three models is shown in Figures 1, 2, and 3. As indicated in Figure 1 , plastics $I,{ }^{a} I I, b$ and $\mathrm{III}^{c}$ represent porcelain, gold alloy, and dentin. Material I was luted to material II with improved dental stone; materials II and III were cemented together with epoxy cement. ${ }^{1}$ The peripheral outline of each section and the dentin are the same for the three designs, as is the thickness of the gold alloy on the labial side of the model. The models differ in the position of the porcelain-fused-to-gold joint; it is placed more to the lingual side from model 1 to model 3.

Compressive loads were applied to a location that represented the cingulum. The models were supported in a brass jig the sides of which are visible in Figures 1 to 3 . The models and the jig contacted at positions indicated by the solid white arrows shown in Figure 1; this represents the con-

a PSM-1, modulus of 340,000 psi and a fringe constant of $40 \mathrm{lbs} / \mathrm{in}^{2} /$ fringe/in. Photolastic, Inc., Malvern, $\mathrm{Pa}$.

b PSM-5, modulus of 450,000 psi and a fringe contant of $60 \mathrm{lbs} / \mathrm{in}^{2} / \mathrm{fringe} / \mathrm{in}$. Photolastic, Inc., Malvern, Pa.

c PL-2, modulus of 100,000 psi and a fringe constant of $37.8 \mathrm{lbs} / \mathrm{in}^{2} /$ fringe in. Photolastic, Inc., Malvern, Pa. 


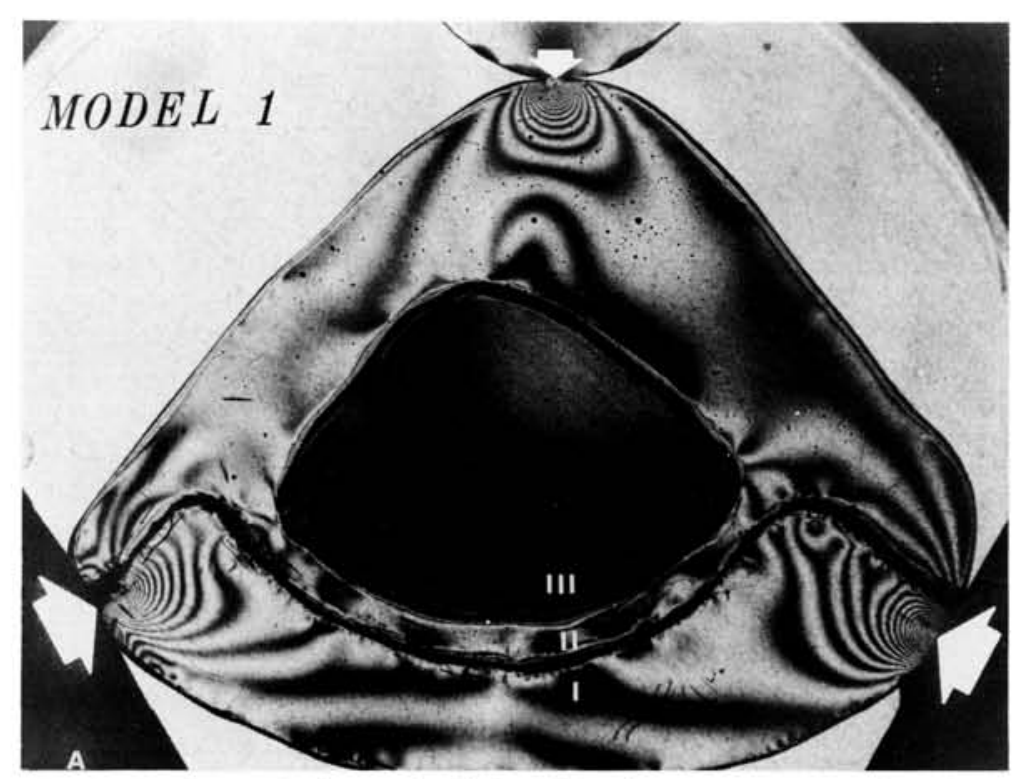

FIG 1.-Isochromatic fringes in light field of transverse composite photoelastic model 1 and 80 pound load. Materials are identified (I, II, and III) .

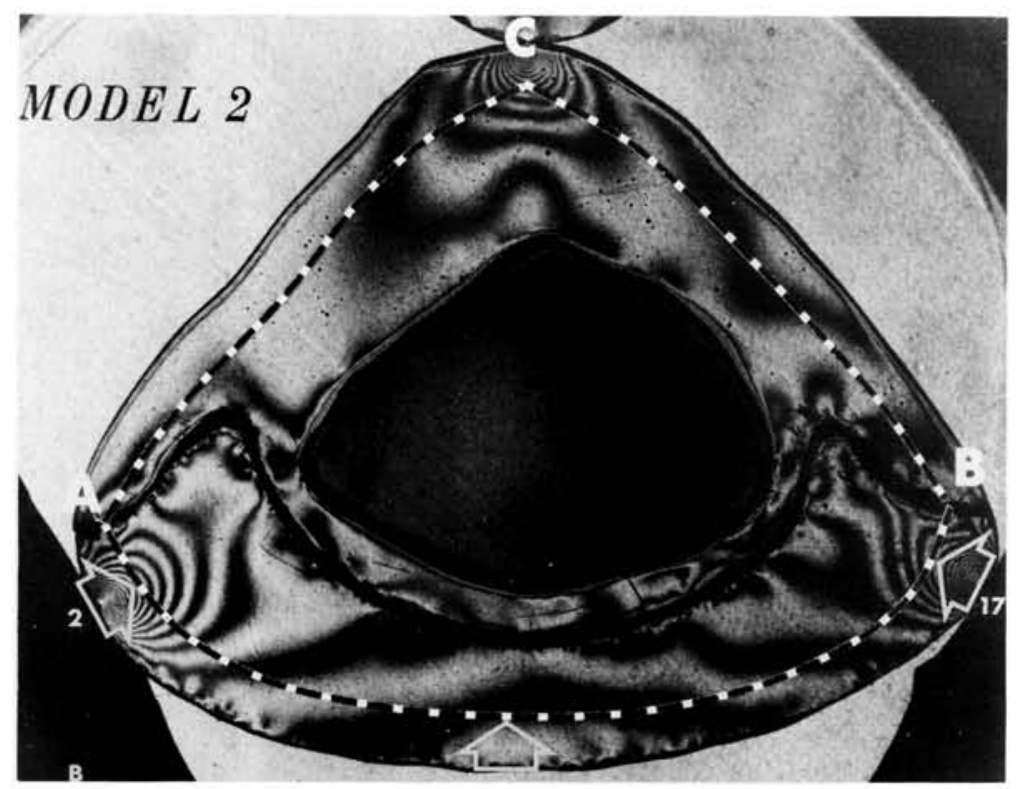

Fig 2.-Isochromatic fringes in light field of transverse composite photoelastic model 2 under 80 pound load. Difference in prinicpal stress was plotted for lines indicated. 


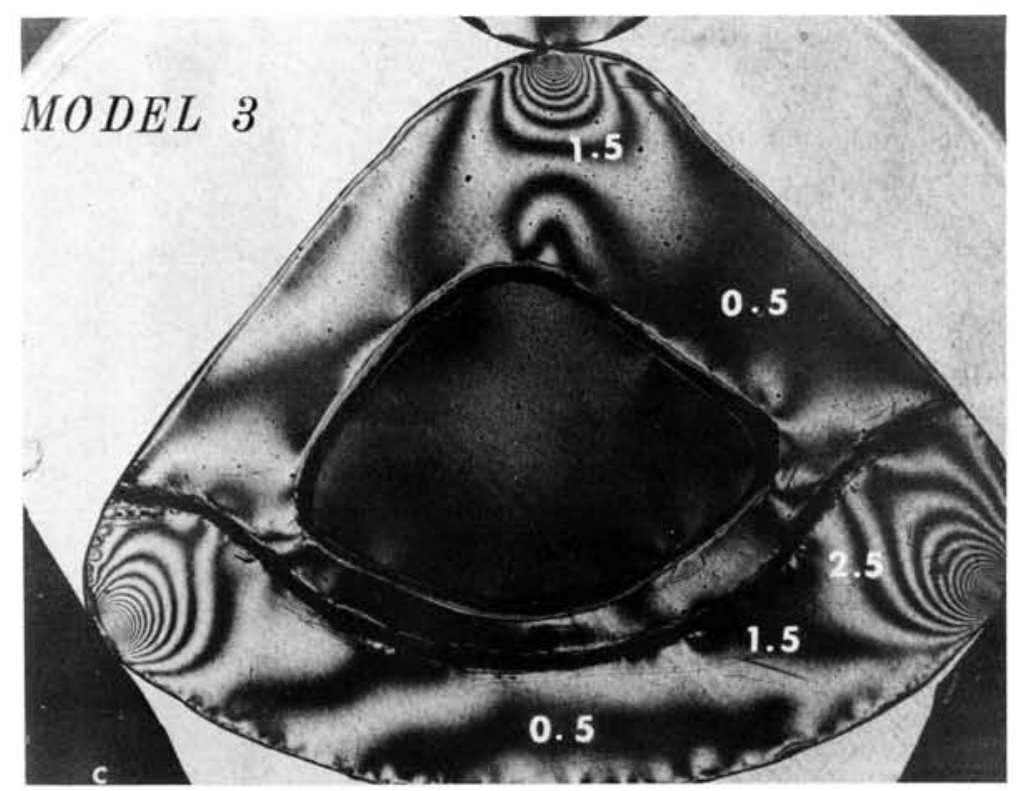

Fic 3.-Isochromatic fringes in light field of transverse composite photoelastic model 3 under 80 pound load. Typical fringe orders as shown.

tact of the restoration with adjacent teeth on the mesial and the distal sides. We realized that the direction of the application of the load is not identical in a model and an actual restoration, and the loading at the contact points is not identical. In spite of differences in the test conditions and the real condition, we believed that the approximations would not significantly alter the overall distribution of stress and that they would allow the design of the models to be compared.

The models were loaded under 40,60 , and 80 pounds and the fringe orders were noted. The order of some of the fringes is indicated in Figure 3. Plots of $\sigma_{1}-\sigma_{2}$, twice the maximum shear stress, were made along lines $A B$ and $C B$, the locations of which are shown in Figure 2. The values for the maximum shear stresses were calculated from the values of the fringe order as described earlier.2

\section{Results}

Visual examination of Figures 1, 2, and 3 showed that the dentin area was essentially under a uniform state of shear stress, and showed a maximum fringe order of 0.5 , which represented a $\sigma_{1} \cdot \sigma_{2}$ value of $75.6 \mathrm{psi}$ and a maximum shear stress value of 37.8 psi. The state of stress differed most, for the three models, in the areas that represented the contact points with adjacent teeth. As expected, the shear stress distribution was generally symmetrical on a vertical line through point $C$ (Fig 2).

The values of the stress, $\sigma_{1}-\sigma_{2}$, along line $A B$ are shown in Figure 4 for the three models. The highest values of $\sigma_{1} \cdot \sigma_{2}$ were for model 1 and the lowest were for model 3 . Selected points along line $A B$ are shown in Figure 2 by the white-outlined arrows. The remaining points indicated in Figure 4 are equidistant from each adjacent point. The lower values of $\sigma_{1}-\sigma_{2}$ near the end of the line segment $A B$ were the result of the porcelain-gold junction being moved toward the lingual side.

The effect of load on $\sigma_{1}-\sigma_{2}$ as a function of position along line $A B$ is shown in Fig. ure 5 for model 2. A regular increase in $\sigma_{1}-\sigma_{2}$ resulted from increasing the load from 40 to 60 to 80 pounds, although greater differences in values occurred at points near the contact points.

The effect of the design on $\sigma_{1} \cdot \sigma_{2}$ along lines $C B$ and $C A$ are shown in Figure 6 . The values of $\sigma_{1}-\sigma_{2}$ obtained at an 80 pound 


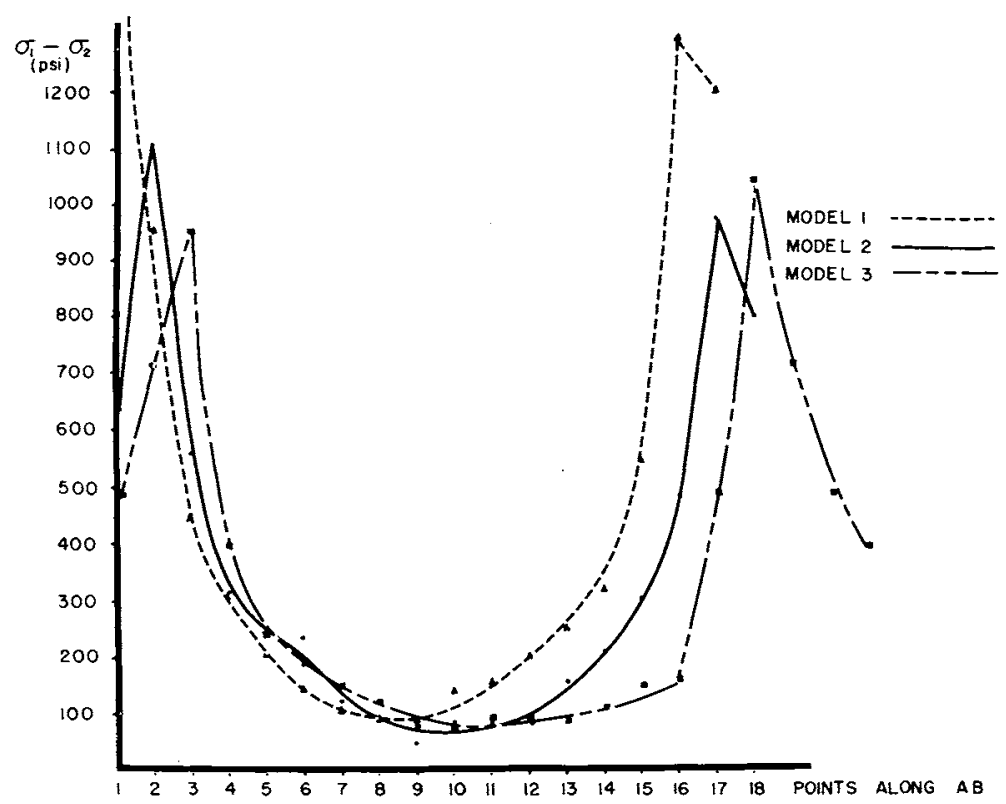

Fig 4.-Difference in principal stresses, $\sigma_{1}-\sigma_{2}$, is plotted for line $A B$ for models 1,2 , and 3 .

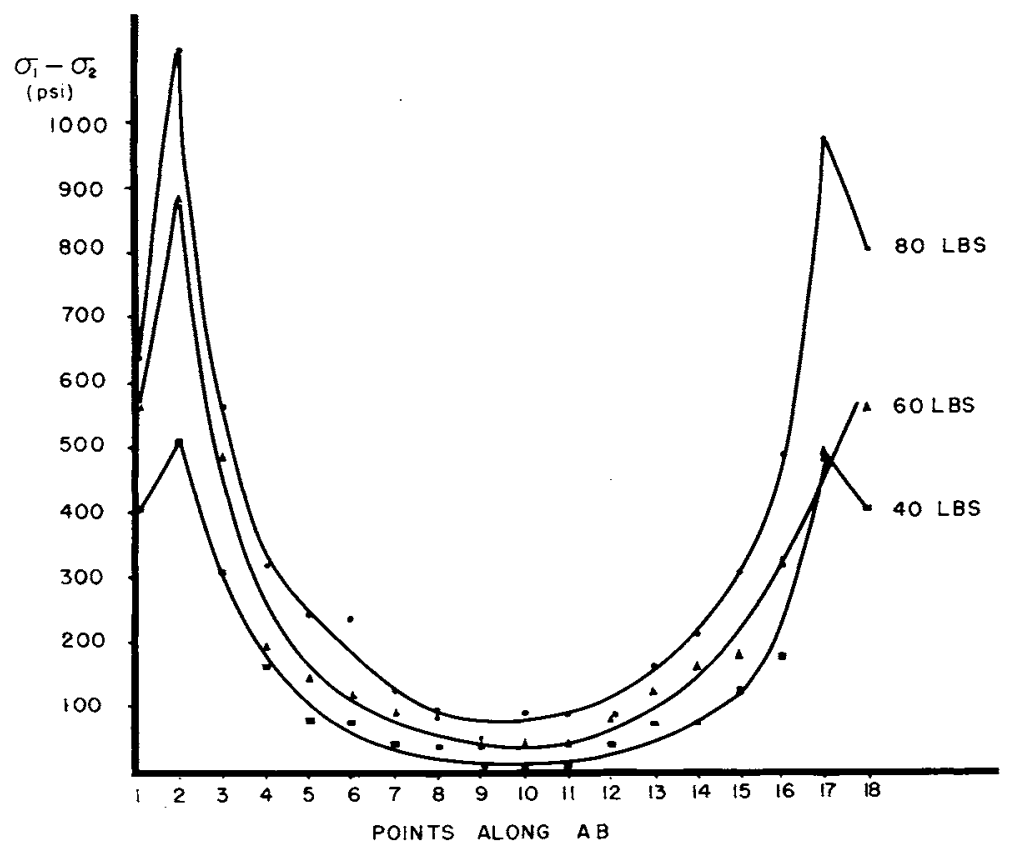

FIG 5.-Difference in principal stresses, $\sigma_{1}-\sigma_{2}$, is plotted for model 2 along line $A B$ at loads of 40,60 , and 80 pounds. 


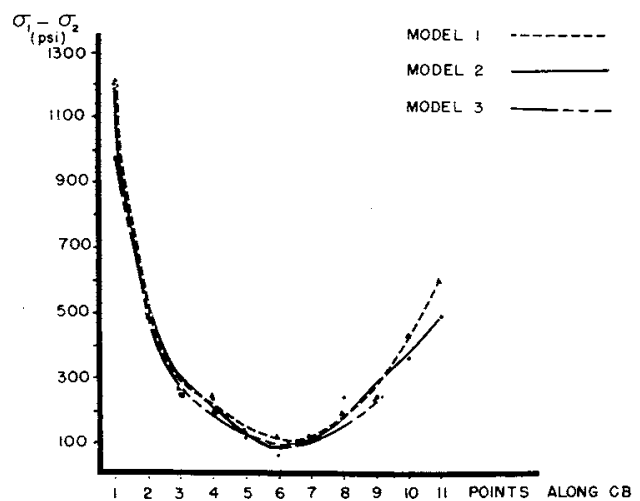

Fig 6.-Difference in principal stresses, $\sigma_{1}-\sigma_{2}$, is plotted along line $C B$ for models 1,2 , and 3 at 80 pound load.

load are shown only along $C B$ since the symmetry of the models resulted in the same curves for $C A$. The highest values for $\sigma_{1} \cdot \sigma_{2}$ were just under the point of loading; they decreased to a minimum value of 100 psi at the midpoint and then increased to about 500 psi as the porcelain-gold junction was reached. No significant differences in $\sigma_{1}-\sigma_{2}$ as a function of position were observed among the three models. A regular increase in $\sigma_{1}-\sigma_{2}$ occurred along $C B$ as the load was increased from 40 to 80 pounds, as shown in Figure 7.

\section{Discussion}

The stress distribution of transverse sections of photoelastic composite models representing porcelain-fused-to-gold restorations and preparations is a substantial confirmation of the results from a previous study of labial-lingual and mesial-distal sections of similar preparations. ${ }^{1}$ The state of stress of the dentin in both studies has been shown to be low. The most important factor in the stress distribution in the models is the position of the porcelain-gold junction with respect to the site of loads. The restoration should be designed so that the porcelain-gold junction is removed from sites of load application; thus, it is recommended that the junction in transverse sections be placed well to the lingual side of the contact points. The optimum design would include placing the porcelain-gold junction midway between the incisal contact point and any contact in the area of the cingulum, and well to the lingual side of the contact areas between ad-

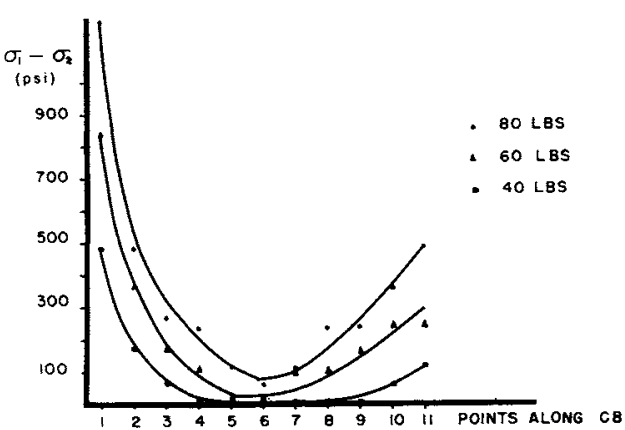

Fig 7.-Difference in principal stresses, $\sigma_{1}-\sigma_{2}$, is plotted along line $C B$ for models 1, 2, and 3 at 40,60 , and 80 pound loads.

jacent teeth. This design would allow for maximum strength with a bulk of gold in the area of the cingulum, and would provide the optimum distribution of stress in the restoration. Under these conditions, low maximum shear stresses would be observed on the labial surface of the porcelain, as shown in Figure 3. The highest stresses in the porcelain would be compressive stresses from incisal loading and somewhat lower stress at the contact areas with adjacent teeth.

\section{Conclusions}

Shear stress distribution measurements on photoelastic models of transverse sections of porcelain-fused-to-gold restorations and preparations showed that low stresses exist in supporting dentin. The optimum design with respect to distribution of the shear stress was the model in which the porcelaingold junction was on the lingual side of the contact areas between adjacent teeth. These data are consistent with results from labiallingual and mesial-distal composite photoelastic models of porcelain-fused-to-gold restorations and preparations.

\section{References}

1. Craig, R.G.; El-Ebrashi, M.K.; and Peyton, F.A.: Stress Distribution in Porcelain-Fusedto-Gold Crowns and Preparations Constructed with Photoelastic Plastics, J Dent Res 50: 1278-1283, 1971 .

2. Craig, R.G.; El-Ebrashi, M.K.; LE Peak, P.J.; and Peyton, F.A.: Experimental Stress Analysis of Dental Restorations. Part I. TwoDimensional Photoelastic Stress Analysis of Inlays, J Prosthet Dent 17: 277-291, 1967. 